Research Paper

\title{
Hypoxia-elevated circELP3 contributes to bladder cancer progression and cisplatin resistance
}

Yinjie Su${ }^{1 *}$, Weiping Yang ${ }^{1,2^{*}}$, Ning Jiang ${ }^{1,4^{*}}$, Juanyi Shi ${ }^{1}$, Luping Chen ${ }^{3}$, Guangzheng Zhong ${ }^{1}$, Junming Bi ${ }^{1}$, Wei Dong1, Qiong Wang1, Chunhui Wang ${ }^{2}$, Tianxin Lin ${ }^{1}$

1. The Department of Urology, Sun Yat-Sen Memorial Hospital, Sun Yat-Sen University, Guangzhou, China;

2. The Department of Urology, Yan 'an Hospital Affiliated with Kunming Medical University, Kunming, China;

3. The Department of Pediatric Surgery, Sun Yat-Sen Memorial Hospital, Sun Yat-Sen University, Guangzhou, China;

4. The Department of Urology, Zhujiang Hospital of Southern Medical University, Guangzhou, China.

${ }^{*}$ Authors who contributed equally to this article.

$\bowtie$ Corresponding authors: Tianxin Lin: tianxinl@sina.com; Tel.: +86-13724008338. Chunhui Wang: 13211604155@163.com; Tel.: +86-13211604155

(c) Ivyspring International Publisher. This is an open access article distributed under the terms of the Creative Commons Attribution (CC BY-NC) license (https://creativecommons.org/licenses/by-nc/4.0/). See http://ivyspring.com/terms for full terms and conditions.

Received: 2018.04.22; Accepted: 2018.08.18; Published: 2019.01.01

\begin{abstract}
Hypoxia plays a critical role in cancer biology. It induces genomic instability, which in turn helps cancer cells respond adaptively to meet the needs of carcinogenesis, cancer progression and relapse. Circular RNA has not been reported among the variety of downstream factors in this adaptive response. Although a few studies have demonstrated the important role of circular RNAs in driving human bladder cancer progression, their carcinogenic roles are still under investigated. Here, we identified a hypoxia-elevated circular RNA, circELP3, that contributes to bladder cancer progression and cisplatin resistance. Decreasing the level of circELP3 via siRNA clearly reduced the in vitro proliferation and cisplatin resistance of bladder cancer cells and promoted apoptosis. Interfering with circELP3 suppressed tumor xenograft growth in nude mice in vivo. In addition, lower circELP3-expressing bladder cancer cells displayed poorer self-renewal capacity, as demonstrated by lower levels of sphere formation and stem cell marker expression. Furthermore, in human bladder cancer patients, strong correlations between a high circELP3 level and advanced tumor grade and lymph node metastasis were observed. In summary, we provide the first direct evidence that circular RNA participates in the adaptive response to hypoxia and may play a role in the progression and drug resistance of bladder cancer.
\end{abstract}

Key words: Hypoxia, Circular RNA, Bladder cancer, Progression, Cisplatin resistance

\section{Introduction}

Bladder cancer is the sixth most common cancer in male patients and has a global incidence of over 400,000 new cases per year, with 165,000 bladder-cancer-related deaths each year [1, 2]. After bladder cancer progresses to the advanced stage, the only option for treatment is cisplatin-combined chemotherapy [3]. Cisplatin is a platinum-based compound that exerts a broad spectrum of anti-tumor activities in various cancers via the formation of both inter- and intra- strand DNA adducts [4]. However, intrinsic or acquired cisplatin resistance severely attenuates its therapeutic potential [5]. The molecular drivers of cisplatin resistance are still largely unknown. Hence, it is vitally important to further identify the molecular mechanisms that aid cancer cells in surviving chemotherapy.

Hypoxia plays a critical role in cancer biology [6]. An adequate degree of hypoxia in the cancer niche promotes cancer progression [7]. During the development of cancer, the conventional blood supply no longer meets the needs of highly proliferative cancer cells; aberrant heteromorphic tissue formation due to unsynchronized cancer growth hampers the normal vessel structure, which eventually leads to a hypoxic tumor environment [8, 9]. Since DNA damage is usually caused by hypoxia, 
cancer cells have evolved an adaptive response to survive under hypoxia [10]. It has been reported that hypoxia enhances cisplatin resistance in cancer cells $[11,12]$ and that the gene instability induced by hypoxia assists cancer cells in passing on the DNA damage caused by cisplatin [13, 14]. These findings suggest that studying hypoxia may shed a new light on the molecular mechanisms of cisplatin resistance.

Circular RNAs are a spectrum of conserved endogenous RNAs that are formed by exon skipping or back-splicing events. They have been shown to play roles in driving cancer behavior [15]. However, among the variety of downstream factors in the adaptive response under hypoxia, no reports have shown the involvement of circular RNA. In human bladder cancer, a few reports have demonstrated that circular RNAs can assist in cancer progression, including circ-ITCH [16], circ-HIPK3 [17] and circ-MYLK [18], but none of these studies focused on cisplatin resistance or hypoxia. Given the promising role of circular RNA in bladder cancer, we are interested in whether endogenous circular RNA can facilitate the progression of bladder cancer. Hence, the objectives of our current study were to molecularly characterize bladder cancer and to investigate the role of circular RNA in the hypoxic adaptive response, which may further elucidate additional mechanisms of cisplatin resistance in bladder cancer. Here, we have identified a hypoxia-elevated circular RNA, circELP3, that contributes to bladder cancer progression and cisplatin resistance. Like most circular RNAs, circELP3 is spliced from its host gene, ELP3. Through back-splicing events between the $11^{\text {th }}$ and $14^{\text {th }}$ exon of ELP3, circELP3 is circularized (Figure $1 G)$. ELP3 is considered to drive the initiation of multiple cancers, while no report has suggested a related function for circELP3. Considering the promising role of circELP3 in regulating bladder cancer biologically and clinically, we provide the first direct evidence that circular RNA participates in the adaptive response to hypoxia and may play a role in the progression and drug resistance of bladder cancer.

\section{Materials and methods}

\section{Human tissue sample preparation}

Bladder cancer tissue and its adjacent normal bladder tissue were obtained from patients diagnosed with bladder cancer at Sun Yat-Sen Memorial Hospital from 2015 Jun 1st to 2017 Mar 5th. A total of 18 pairs of tissue samples and 30 bladder cancer samples were collected in liquid nitrogen and stored at $-80^{\circ} \mathrm{C}$ until RNA extraction. The use of these tissue samples was approved by the ethics committee of Sun Yat-Sen University. All patients signed a contract for use of their tissues in research.

\section{Cell culture}

Human bladder cancer cell lines (T24 and 5637) were purchased from ATCC. T24 and 5647 cells were cultured in 1640 medium (Gibco, USA) supplemented with 10\% fetal bovine serum (FBS) (Biological Industries, USA) and $100 \mathrm{U} / \mathrm{ml}$ penicillin and streptomycin (Gibco, USA). Cells were cultured at $37^{\circ} \mathrm{C}$ in a humidified incubator (Thermo, Germany) containing $5 \% \mathrm{CO}_{2}$. For hypoxia, cells were cultured under $1 \% \mathrm{O}_{2}$ and $5 \% \mathrm{CO}_{2}$ in a $37^{\circ} \mathrm{C}$ humidified incubator (Smartor 118pro, China innovation instrument, Ningbo, China) with 1640 medium supplemented with $10 \%$ FBS and $100 \mathrm{U} / \mathrm{ml}$ penicillin and streptomycin.

\section{RNA preparation and quantitative real-time PCR}

Total RNA was extracted using an RNAiso Plus kit (TaKaRa, Japan) and reverse-transcribed using a PrimeScript $^{\mathrm{TM}}$ RT Master Mix kit (TaKaRa, Japan). Quantitative real-time PCR analysis (Light Cycler 480 Roche, German) was performed to detect the expression of ELP3 and circELP3 using a SYBR Green kit (TaKaRa, Japan). The related PCR primers were as Table 1.

Table 1. List of PCR primers

\begin{tabular}{|c|c|c|}
\hline & Forward & Reverse \\
\hline circELP & 5'-AGTACGAGAGCTGCATGTGT-3 & 5'-TGCCAGCTCTCTCAGGTTAC- \\
\hline 3 & $x^{\prime}$ & $3^{\prime}$ \\
\hline \multirow[t]{2}{*}{ ELP3 } & $5{ }^{\prime}-$ & $5^{\prime}-$ \\
\hline & CAGTGAGGGCCTTGATTCCA-3' & TGAGCTTCATTCAACGGAGC-3' \\
\hline \multirow[t]{2}{*}{ Sox2 } & $5^{\prime}-$ & 5'- GCTGATCATGTCCCGTAGGT \\
\hline & GCCCTGCAGTACAACTCCAT-3' & $-3^{\prime}$ \\
\hline \multirow[t]{2}{*}{ Nanog } & $55^{\prime}$ & 5'-CTGGTGGTAGGAAGAGTAA \\
\hline & TGCTGAGCCCTTCTGAATCA-3' & AGG-3' \\
\hline \multirow[t]{2}{*}{ Oct4 } & $5^{\prime}-$ & $5^{\prime}-$ \\
\hline & GCAGCGACTATGCACAACGA-3' & $\begin{array}{l}\text { CCAGAGTGGTGACGGAGACA } \\
-3^{\prime}\end{array}$ \\
\hline \multirow[t]{2}{*}{ DHX9 } & $5^{\prime}-$ & 5'- CCAACACCTGCACCATATCC \\
\hline & TCTAGTTACAGTGGTGGAGGC-3' & $-3^{\prime}$ \\
\hline \multirow[t]{2}{*}{ ADAR1 } & $5^{\prime}-$ & 5'-CAGCAAAGCCATGGGAGC \\
\hline & GAATCCATGATGCCCAACAAG-3 & $-3^{\prime}$ \\
\hline \multirow[t]{2}{*}{ QKI } & $5^{\prime}-$ & $5^{\prime}-$ \\
\hline & GAACGGAGAACTGCATGACC-3' & $\begin{array}{l}\text { GGTGGAGGATAAGCAGCACT } \\
-3^{\prime}\end{array}$ \\
\hline
\end{tabular}

\section{RNase $\mathbf{R}$ treatment and agarose gel electrophoresis}

For RNase R treatment, $1 \mu \mathrm{g}$ of extracted RNA was treated with $3 \mathrm{U}$ of RNase R (Epicenter, USA) at $37^{\circ} \mathrm{C}$ for 1 hour. The sample was mixed with $50 \mu \mathrm{l}$ of phenol-chloroform and stored at $-70^{\circ} \mathrm{C}$ overnight for better sedimentation. Later, $500 \mu \mathrm{l}$ of isopropanol was added, and the samples were centrifuged at 12000 $\mathrm{rpm}$ at $4^{\circ} \mathrm{C}$ for $15 \mathrm{~min}$; the supernatant was removed and washed twice with $75 \%$ ethanol. For agarose gel electrophoresis, $5 \mu \mathrm{l}(50 \mathrm{ng})$ of prepared cDNA was 
run on a $1 \%$ agarose gel and developed in a G BOX F3 (Thermo, German).

\section{RNA in situ hybridization}

The circELP3 FISH probes were designed and synthesized by Genepharm (Suzhou, China). The signal of the circELP3 FISH probe was detected according to the instructions provided in a Fluorescence In situ Hybridation kit (RiboBio, Guangzhou, China).

\section{Cell transfection}

The siRNAs for circELP3 and NC were purchased from Genepharm (Suzhou, China). Cells were seeded at a density of $50 \% 24$ hours in advance. During transfection, $3 \mu \mathrm{g}$ of iMAX (Lipofectamine RNAi MAX Reagent, Invitrogen, USA) and $5 \mu \mathrm{g}$ of siRNA were added to $200 \mu \mathrm{l}$ of Opti-MEM (Gibco, USA) according to the manufacturer's guide. Cells were collected for further experiments after 48 hours of transfection. Two siRNAs were designed for circELP3 with the following sequences:

siRNA-1: 5'-GCUGUGAUAUCAGGGAUAUTT-3'; siRNA-2: 5'-GAUAUCAGGGAUAUUCCAATT-3'.

According to the siRNAs sequence, 2 shRNAs were constructed and cloned into a GV248 vector (GENECHEM, China).

\section{CCK8 assay}

Cells were harvested and plated in 96-well plates overnight at a concentration of 1000 cells per well. Cell viability was measured after culture from the first day to the fifth day. Briefly, CCK8 (Beyotime, China) was added to the culture medium to reach a concentration of $10 \%$. After 2 hours of incubation, the absorbance of the supernatant in each well at $450 \mathrm{~nm}$ was measured using a microplate reader. For the cisplatin treatment, cells were treated with cisplatin (Sigma, USA) at concentrations of $5 \mu \mathrm{M}, 10 \mu \mathrm{M}, 20$ $\mu \mathrm{M}, 40 \mu \mathrm{M}$ and $80 \mu \mathrm{M}$. Cell viability was detected after 24 hours.

\section{Cell clone formation assay}

Cells were harvested and plated in 6-well plates overnight at a concentration of 200 cells per well and grown in medium with $10 \%$ FBS. After 2 weeks of culture, cells were stained with $0.1 \%$ crystal violet, and colonies that contained $>50$ cells were counted. For cisplatin treatment, cells were treated with cisplatin at a concentration of $5 \mu \mathrm{M}$. Cell clones were counted after 2 weeks of culture.

\section{Apoptosis assay}

More than $3 \times 10^{5}$ cells per sample were prepared and stained with reagents from an Annexin V-FITC/PI Apoptosis Detection kit (CWBio, China).
According to the manufacturer's instructions, cells were eventually analyzed via flow cytometry.

\section{Sphere formation assay}

Cells were seeded at a density of 1-5 cells/well in ultralow-attachment 96-well plates and grown in serum-free medium supplemented with insulin, EGF and $\beta$-FGF (Sigma, USA). After culturing for 10 days, colonies that contained $>20$ cells were counted.

\section{Animal studies}

All the animal studies were approved by the Animal Management Committee of Sun Yat-Sen University. Ten nude male mice (3-4 weeks old) were used in this study. To imitate the in vivo effect of carcinogenesis, approximately $10^{7} \mathrm{~T} 24$ cells (Vector and sh-circELP3) were injected subcutaneously. Width (W) and length (L) of tumors was measured each week using calipers. The volume $(\mathrm{V})$ was calculated via the formula $\mathrm{V}=(\mathrm{W} 2 \times \mathrm{L}) / 2$. After 3 weeks of injection, the mice were sacrificed. The tumors were fixed in $4 \%$ formalin, and tumor weights were measured.

\section{Statistical analysis}

Statistical analysis was performed using GraphPad 6.0. Correlations between the independent groups were analyzed using a t-test, and one-way ANOVA was used to evaluate the significance of differences between multiple groups. The results were considered statistically significant when $p<0.05$ or $p$ $<0.01$.

\section{Results}

\section{Characterization of circELP3 and its elevation under hypoxia.}

Two pairs of bladder cancer and adjacent normal tissues were analyzed using high-throughput RNA-seq. Only 567 circular RNAs were significantly dysregulated among the 6834 identified circular RNAs (Figure 1A). In addition, using quantitative real-time PCR, the 20 most upregulated, 20 most downregulated and 20 randomly selected circular RNAs were analyzed, and only circELP3 was obviously upregulated (2.56 \pm 0.32 -fold in T24 cells and $2.79 \pm 0.15$-fold in 5637 cells) under hypoxia (Figure 1B, C). Due to the circular structure, circular RNAs are thought to be more stable than other types of linear RNAs in the face of multiple exonucleases. Hence, we performed RNAse $\mathrm{R}$ treatment to characterize circELP3. After treatment with RNAse R, the linear form of circELP3, ELP3 was considerately digested, while circELP3 was more stable (Figure 1E). Furthermore, based on agarose gel electrophoresis, only the expression of circELP3 was lost in human 
genomic DNA (Figure 1D). These results suggest that the molecule is circular RNA. Furthermore, RNA function strongly relies on cellular location, and circular RNAs in the cytoplasm usually play the role of ceRNAs, while circular RNAs in the nucleus are obligated to regulate protein coding or transcription. Therefore, a florescence in situ hybridization assay was performed and showed that the circELP3 signal is primarily present in the cytoplasm. This result suggests that circELP3 may exert its role via ceRNA activity, for example, as a microRNA sponge (Figure $1 \mathrm{~F})$.

\section{Hypoxia elevates circELP3 independently from the HIF-related pathway or an improved RNA circulation}

A majority of mechanisms contribute to circular RNA elevation, while upregulation of host genes and increased circular RNA circulation are considered the major contributors. hif1a and hif2a are two important transcription factors that cause upregulation of various genes under hypoxia. Hence, to further evaluate the mechanism by which circELP3 is elevated under hypoxia, we first detected whether the elevated circELP3 is related to ELP3 alterations. It was found that under hypoxia, no obvious changes were observed in ELP3 expression (Figure 2A), and the hypoxia-induced elevated circELP3 could not been reduced by hif1a and hif2a interference (Figure 2B, C). These results suggest that elevated circELP3 could not be ascribed to ELP3 alteration or the HIF-related pathway. Next, we detected whether the elevated circELP3 was related to an increased level of circELP3 circulation. DHX9, ADAR1 and QKI are 3 well-known proteins that are able to assist circular RNA circulation [19]. Increased activity of these 3 proteins significantly increases the circulation level. Under hypoxia, it was found that DHX9, ADAR1 and QKI were elevated, with DHX9 upregulated 8.03 \pm 2.44 -fold in T24 cells and 9.45 \pm 3.09 -fold in 5637 cells, ADAR1 upregulated $8.62 \pm 1.98$-fold in T24 cells and 9.11 \pm 3.65 -fold in 5637 cells, and QKI upregulated $4.38 \pm 1.45$-fold in T24 cells and 13.17 \pm 2.95 -fold in 5637 cells (Figure 2D, E, F). However, after silencing the expression of DHX9, ADAR1 and QKI under hypoxia, the elevated circELP3 was not reduced (Figure 2G, H, I)). Hence, all the data above suggest that neither the HIF-associated pathway nor increased circulation contribute to circELP3 elevation.
A

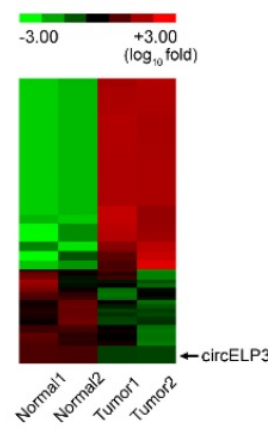

B

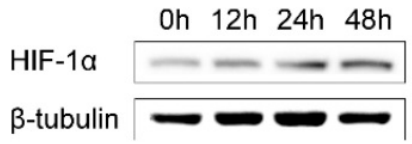

T24

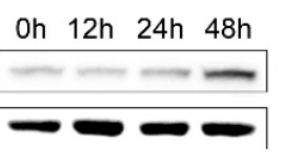

5637

D
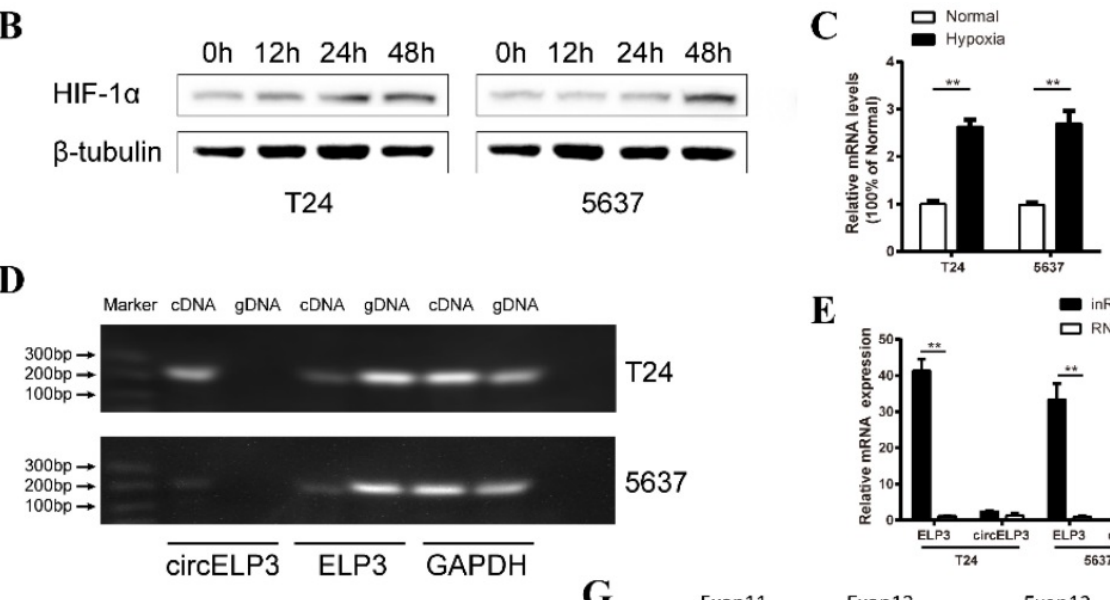

$\mathbf{E}$

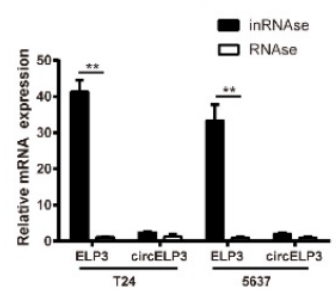

F
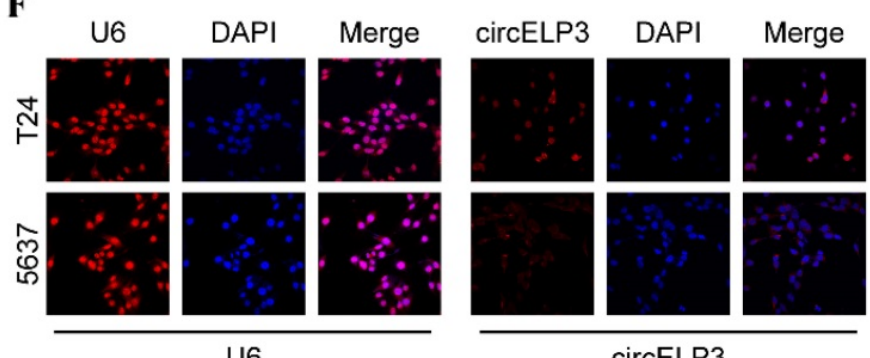

circELP3

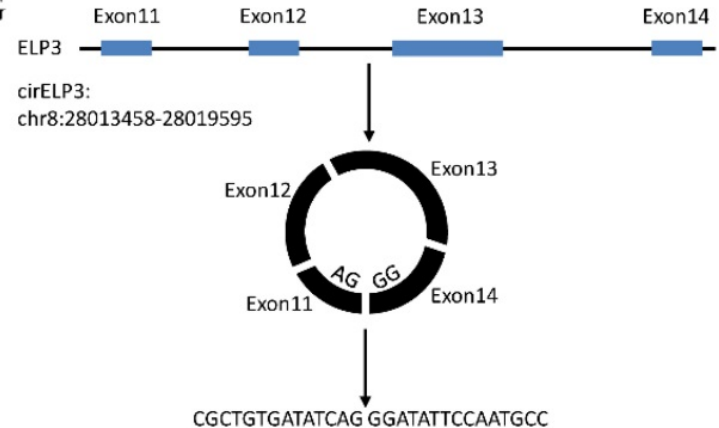

Figure 1: Characterization of hypoxia-elevated circELP3. A: Heat map showing differently expressed circular RNA in 2 sets of paired bladder cancer tissues. Each row represents the expression profile of a tissue sample, and each column corresponds to a specific circular RNA. Upregulated circular RNAs are shown in red, and downregulated circular RNAs are shown in blue. B: Cells cultured with 1\% oxygen, showing that Hif-la increased gradually under hypoxia in T24 and 5637 cell lines, which suggests that the in vitro hypoxic model was established successfully. Among various upregulated circular RNAs, $\mathbf{C}$ shows a significant upregulation of circELP3 under hypoxia. D: Agarose gel electrophoresis shows that the primer for linear circELP3 can amplify ELP3 in both genomic DNA and cDNA but that the primer for the circular form can only amplify ELP3 in cDNA. E: After being treated with RNase R, linear circELP3 was substantially digested, while circELP3 exhibited few differences. F: A florescence in situ hybridization assay was performed to detect the location of circELP3. U6 was used as the positive reference for nuclear location. G: Schematic illustration shows the circulation of circELP3. Scale bars: $\mathrm{F}, 50 \mu \mathrm{M}$. 
A B

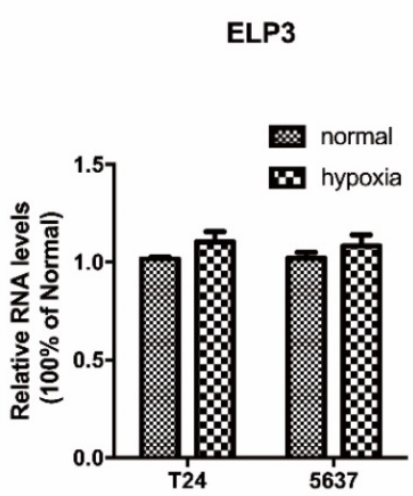

D

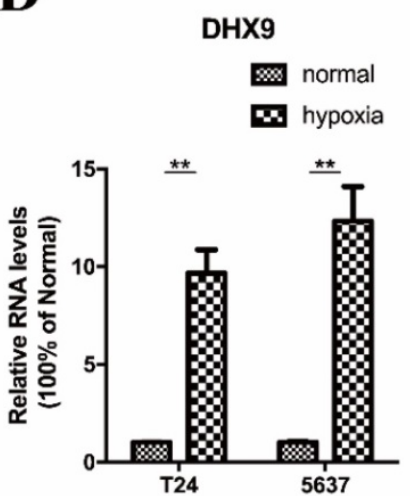

G

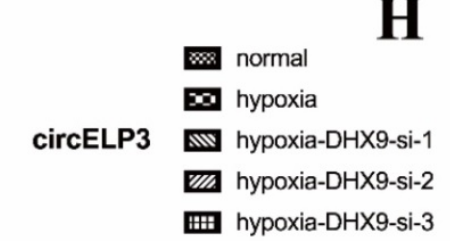

$\mathbf{E}$

H
B

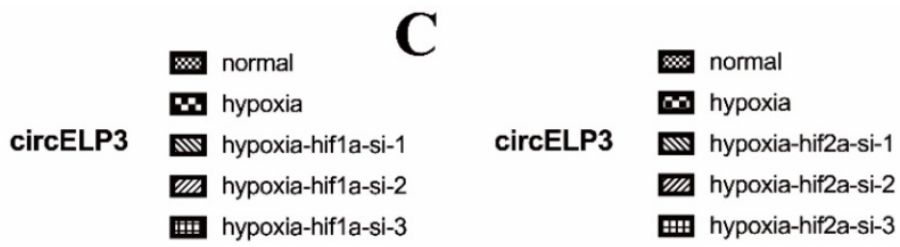

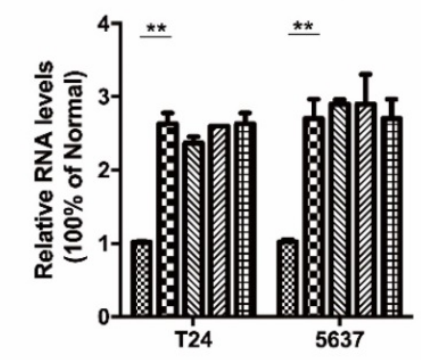

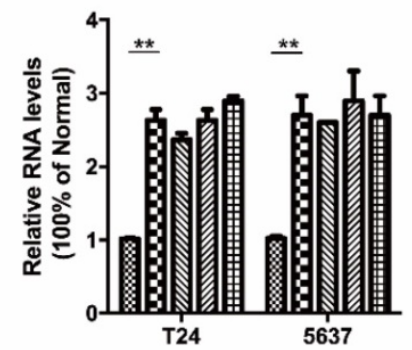

ADAR1

mormal

F

$\infty$ hypoxia
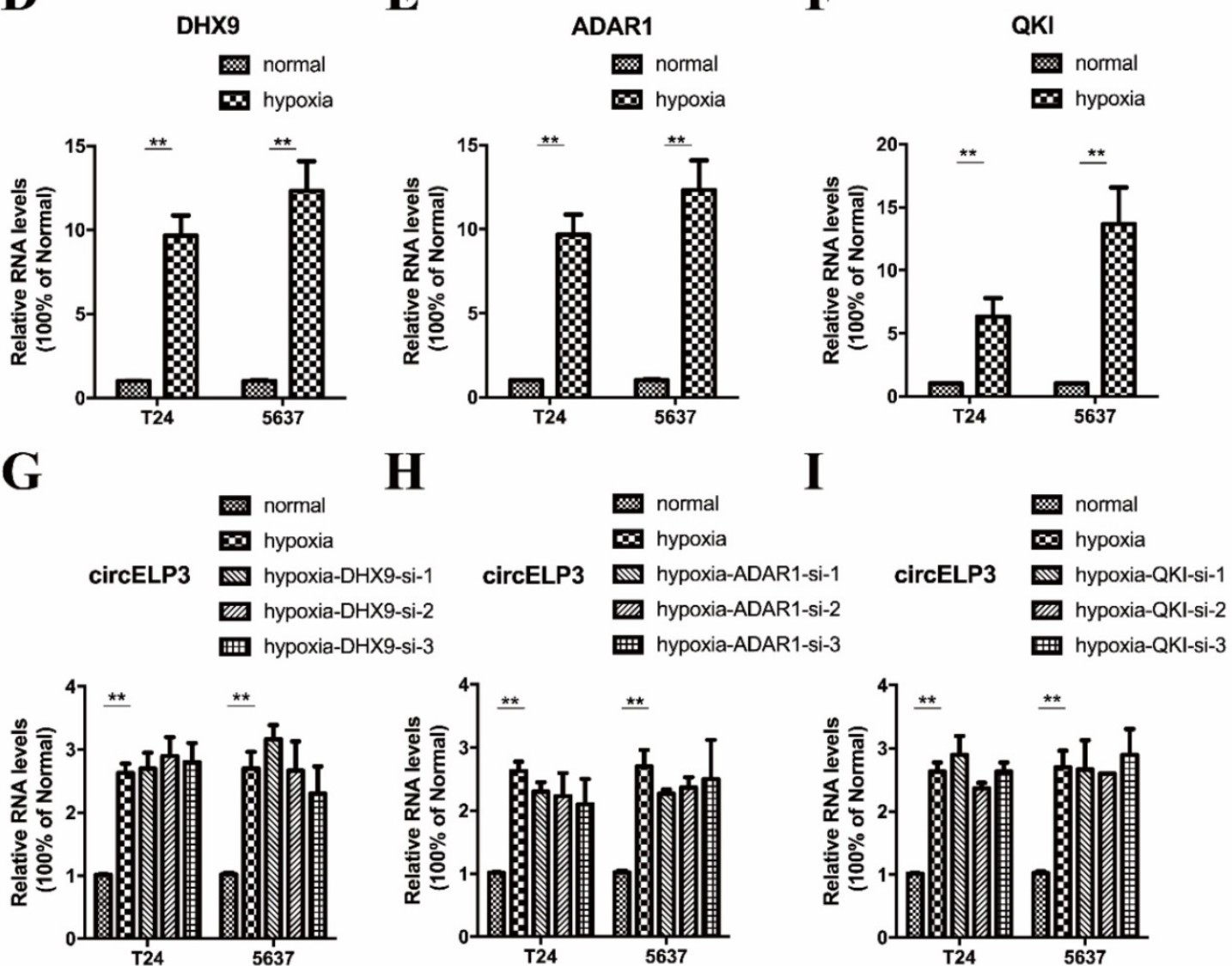

Figure 2: Hypoxia elevates circELP3 independently from the HIF-related pathway or an improved RNA circulation. Quantitative real-time PCR was used to determine the expression of ELP3, circELP3, DHX9, ADAR1 and QKI in different groups. A: Expression of ELP3 between hypoxia and normal conditions; B-C: Effect of hifla and hif2a silencing on circELP3 expression under hypoxia; D-F: Effect of hypoxia on the expression of DHX9, ADAR1 and QKI; G-I: Effect of DHX9, ADAR1 and QKI silencing on circELP3 expression under hypoxia.

\section{Hypoxia-elevated circELP3 promotes proliferation but reduces apoptosis of bladder cancer cells}

Cellular phenotype alteration is direct evidence of altered gene function. Cell survival largely depends on a harmonious balance between cell proliferation and apoptosis. Under hypoxia, appropriate stimulation enhances cancer cell proliferation.
Therefore, in order to determine the function of circELP3, we investigated proliferation as the first step. First, we examined the interference efficiency of siRNA and shRNA toward circELP3 expression in bladder cancer cells. After treatment with siRNA and shRNA, the level of circELP3 was significantly reduced (Figure $3 \mathrm{~A}, \mathrm{~B}$ ). 
A

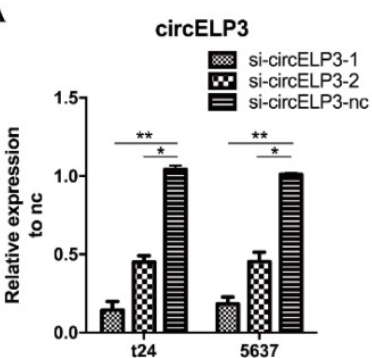

C

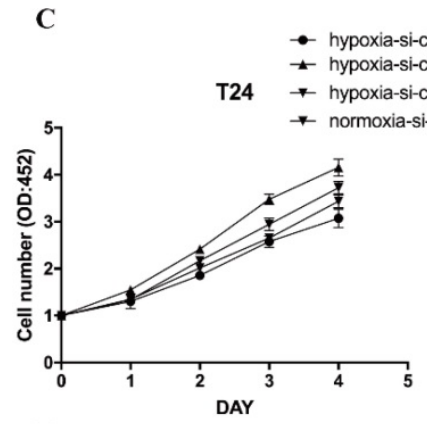

E

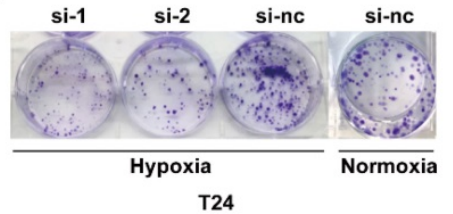

H
B
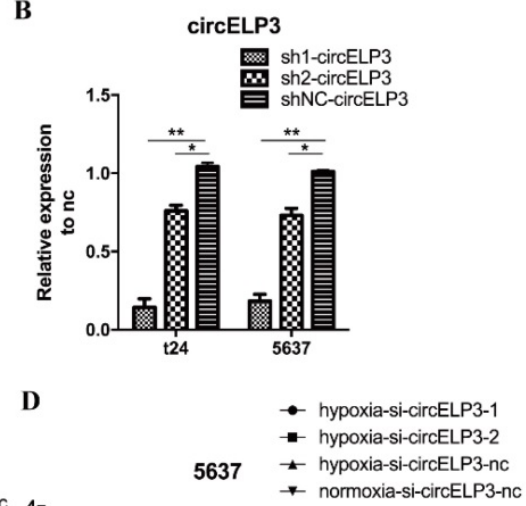

G

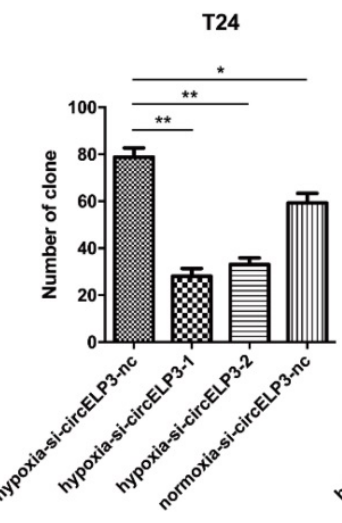

J

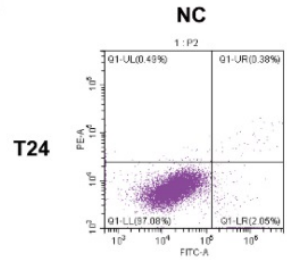

5637 (1)

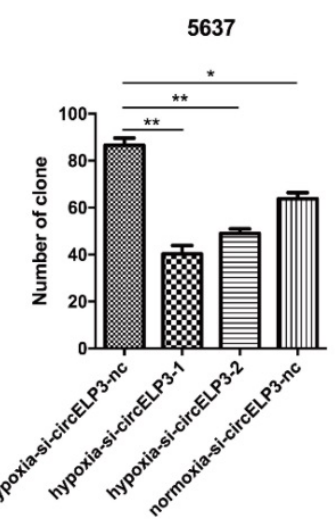

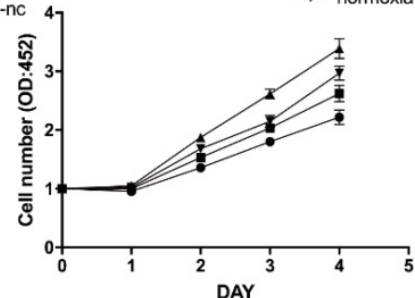

F

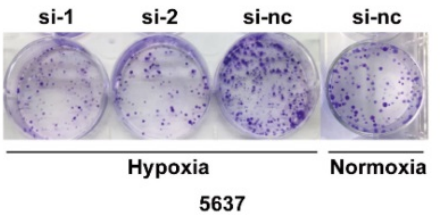

hypoxia-si-circELP3-nc

hypoxia-si-circELP3-1

hypoxia-si-circELP3-2

血 normoxia-si-circELP3-nc
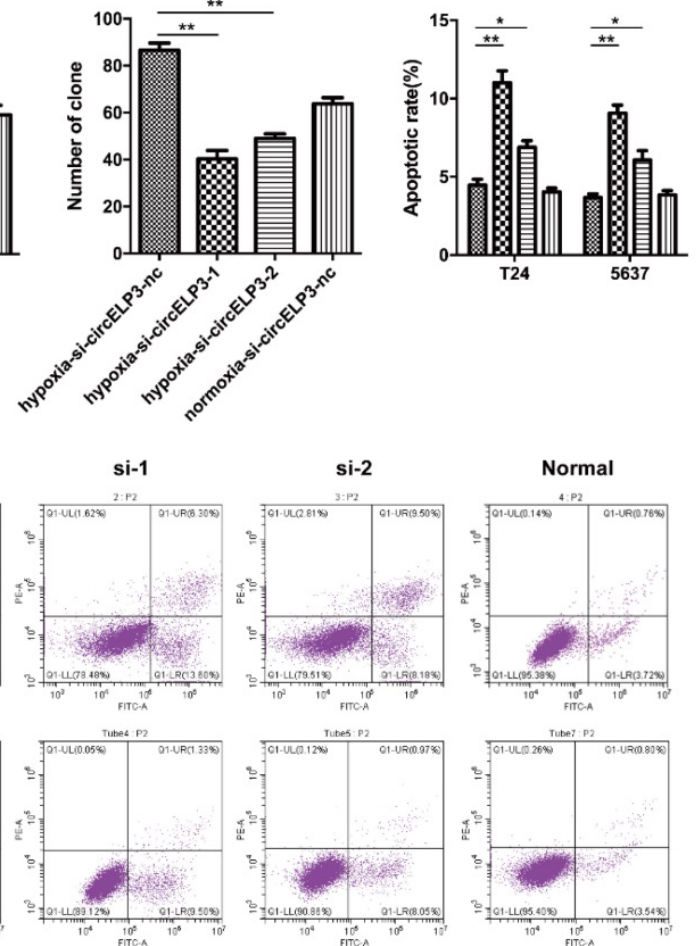

Hypoxia

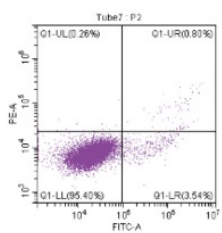

Normoxia

Figure 3: Hypoxia-elevated circELP3 promotes proliferation but reduces apoptosis of bladder cancer cells. A, B: Quantitative real-time PCR was used to detect the circELP3 silencing efficiency of siRNA and shRNA in bladder cancer cells; C, D: CCK8 assays were performed to evaluate effect of circELP3 on cell viability in T24 (A) and 5637 (B) cell lines; E, F: Cell clone formation assays were performed to evaluate the effect of circELP3 on the self-renewal ability of T24 and 5637 cell lines, and G and $H$ show a statistically significant difference in clone formation rate in each treatment group. J: An Annexin V/PI apoptosis assay was used to evaluate the effect of circELP3 on apoptosis in T24 and 5637 cell lines, and I shows a significant difference in the apoptotic rate in each treatment group. 
In addition, by examining proliferation, we found that hypoxia significantly increased the cell viability and clone formation ability of bladder cancer cells. Silencing circELP3 in bladder cancer cells with siRNA apparently reduced the viability of both T24 and 5637 cells after hypoxia treatment (Figure 3C, D). In addition, silencing circELP3 also decreased the number of clones, by $4.21 \pm 0.36$-fold in T24 cells and 1.79 \pm 0.42 -fold in 5637 cells (Figure 3E, F, G, H). For apoptosis, an Annexin V/PI apoptosis assay showed that no significant apoptotic phenotype was induced by hypoxia (Figure 3J), while decreasing circELP3 in bladder cancer cells increased the percentage of apoptotic cells $1.53 \pm 2.87$-fold in T24 cells and 1.35 \pm 2.41 -fold in 5637 cells under hypoxia (Figure 3I). Hence, these data showed that hypoxia-elevated circELP3 can regulate cell viability by promoting proliferation and reducing apoptosis.

\section{Hypoxia-elevated circELP3 promotes cisplatin resistance in bladder cancer cells}

As mentioned, a hypoxic tumor microenvironment may facilitate cisplatin resistance in cancer cells. Thus, we sought to identify whether hypoxia-elevated circELP3 has a role in regulating cisplatin resistance. Therefore, we performed a CCK8 assay with cisplatin concentrations of $5 \mu \mathrm{M}, 10 \mu \mathrm{M}, 20$ $\mu \mathrm{M}, 40 \mu \mathrm{M}$ and $80 \mu \mathrm{M}$, and we observed significantly reduced cell viabilities in the siRNA treatment group compared with the control group after 24 hours under hypoxia (Figure 4A, B). Furthermore, a cell clone formation assay was employed using the concentration $(5 \mu \mathrm{M})$ that produced the best results in the CCK8 assay. After cells were cultured for 2 weeks, reduced clone numbers were found after treatment with siRNA (Figure 4C, D). The reduced clone numbers were statistically analyzed, and a $3.13 \pm 0.26$-fold reduction in T24 cells and $5.03 \pm 1.34$-fold reduction in 5637 cells was found under hypoxia (Figure 4E, F). Taken together, these results suggest that hypoxia-elevated circELP3 can promote cisplatin resistance in bladder cancer cells.

\section{Hypoxia-elevated circELP3 may facilitate cisplatin resistance by targeting cancer stem-like cells}

Emerging evidence has suggested that hypoxia facilitates cancer progression by regulating the stemness of cancer cells. Moreover, several reports suggest that cancer stem-like cells contribute to cisplatin resistance and the progression of bladder cancer. Based on the role of circELP3 in cisplatin resistance, we found that silencing circELP3 with
siRNA significantly reduced the sphere-forming ability of bladder cancer stem-like cells under hypoxia (Figure 5A, B). In addition, circELP3 expression was significantly higher $(5.21 \pm 0.39$ fold $)$ among sphere-forming cells (Figure 5C). Quantitative real-time PCR showed lower levels of the stemness-related genes Oct4, with $2.05 \pm 0.77$-fold less in T24 cells and 1.93 \pm 0.56 -fold less in 5637 cells (Figure 5J); Sox2, with $0.78 \pm 0.21$-fold less in T24 cells and $0.49 \pm 0.38$-fold less in 5637 cells (Figure $5 \mathrm{H}$ ); and Nanog, with no significant difference in expression, after circELP3 silencing under hypoxia (Figure 5I). Further, to identify whether circELP3 could facilitate cisplatin resistance by targeting cancer stem-like cells, we compared the clone formation ability between sphere-forming cells and non-sphere-forming cells after treatment with $5 \mu \mathrm{M}$ cisplatin. Two weeks later, it was found that compared with non-sphere-forming cells, the clone number was insignificantly reduced in sphere-forming cells, with decreased circELP3 expression in both T24 cells (Figure 5D, F) and 5637 cells (Figure 5E, G) under hypoxia. However, our collected sphere cells may not be true cancer stem cells but may simply exhibit a mass with a higher portion of cancer stem cells. Hence, further research is needed to isolate cancer stem cells more accurately and verify the mechanisms among hypoxia-elevated circELP3, cisplatin and cancer stem cells. In all, these data suggest that hypoxia-elevated circELP3 may facilitate cisplatin resistance by targeting cancer stem-like cells.

\section{Interfering with circELP3 suppresses tumor xenograft growth in nude mice}

According to the silencing efficiency of 2 previously constructed shRNAs, sh2-circELP3 displays fainter interfering effect than sh1-circELP3 (Figure 3B). Hence, to evaluate the effect of circELP3 on cancer progression in vivo, we subcutaneously injected the sh1-circELP3 T24 cells into nude mice. Volumes of tumor xenografts were measured weekly. The results showed that circELP3 downregulation significantly suppressed tumor xenograft growth in nude mice compared with the circ-control group $(\mathrm{p}=$ 0.005 , Figure $6 \mathrm{~A}, \mathrm{~B}$ ). Three weeks later, all the mice were sacrificed, and the weights of tumor xenografts were measured. The average weight of the tumor xenografts was obviously less in the sh-circELP3 group than in the control group ( $p<0.05$, Figure $6 C$ ). The results suggest that interfering with circELP3 suppresses tumor xenograft growth in nude mice and were consistent with those in vitro. 
A

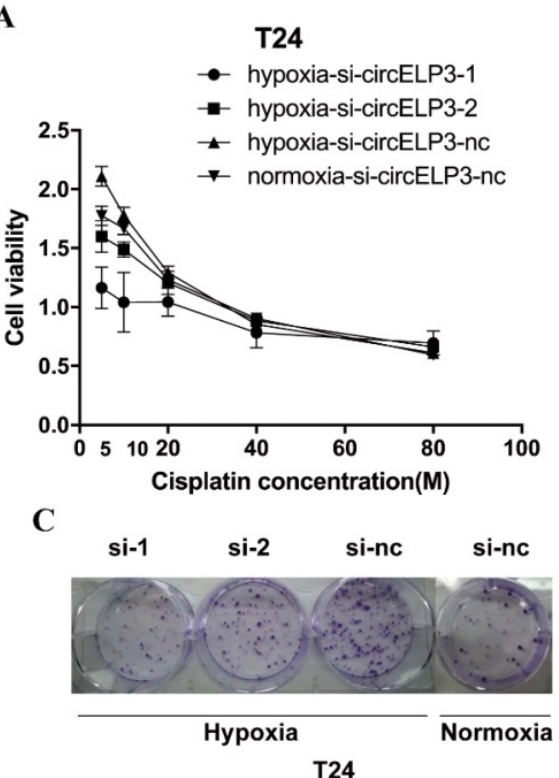

E

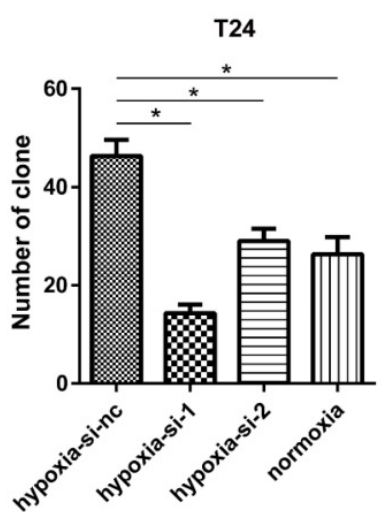

B

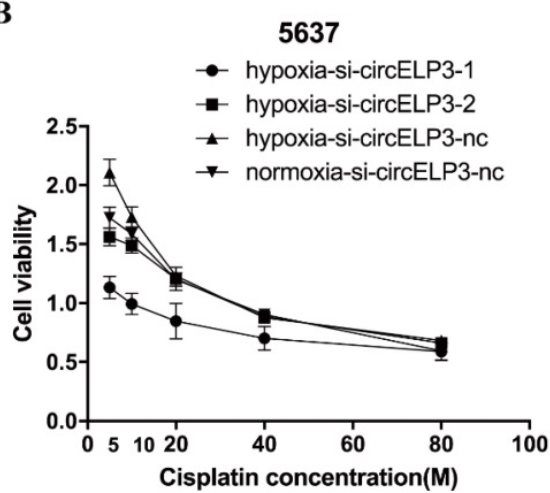

D

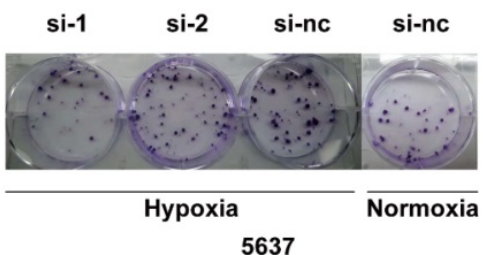

F

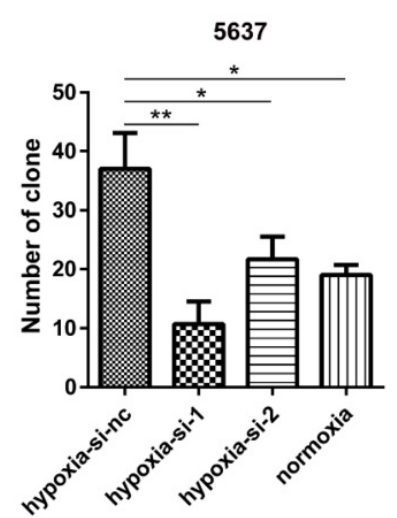

Figure 4: Hypoxia-elevated circELP3 promotes cisplatin resistance in bladder cancer cells. A, B: CCK8 assay to evaluate the effect of circELP3 on the viability of T24(A) and 5637(B) cell lines after treatment with different concentrations of cisplatin; C, D: Cell clone formation assay to evaluate the effect of circELP3 on the self-renewal ability of T24 and 5637 cell lines treated with $5 \mu \mathrm{M}$ cisplatin; E, F: Statistically significant clone numbers in each treatment group.

\section{The clinical relevance of circELP3 in bladder cancer}

We then performed quantitative real-time PCR to measure the clinical relevance of circELP3 in bladder cancer. Although few differences were observed from our microarray test, we still found a strong clinical relevance of circELP3 in bladder cancer. In 18 pairs of bladder cancer and their adjacent tissues, we observed a strong upregulation of circELP3 in cancer tissues; the level of circELP3 was elevated $8.34 \pm 1.07$-fold in bladder cancer compared with the adjacent normal tissue (Figure 7A). Moreover, 30 bladder cancer patients were also analyzed. A higher circELP3 level was significantly correlated with advanced tumor type, i.e., for patients with either a tumor stage higher than T2 (Figure 7B) or lymph node metastasis (Figure 7C), circELP3 expression was upregulated, with $\mathrm{p}<0.05$ for both. However, we did not observe any significant difference between high and low grade bladder cancers (Figure 7D).

\section{Discussion}

Tumor progression relies on intimate cooperation between intercellular and external responses. Despite internal genomic and epigenomic activities, cancer cells must adapt to respond to outside stimulation [20]. Hypoxia constitutes a major part of the tumor microenvironment [21]. Moderate hypoxia assists in the malignant transformation of cancer, which leads to cancer progression [8]. Under hypoxia, several molecules known as hypoxiaresponsive factors are transcribed swiftly for the hypoxia-adaptive response, such as hifla and hif2a [22]. As transcriptional factors, hif1a and hif2a act on the hypoxia-responsive element of downstream genes, which in turn leads to an alternative genomic landscape and contributes to cancer progression. We call this the hypoxic adaptive response. 
A

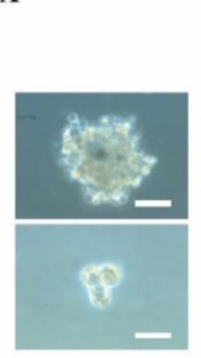

B

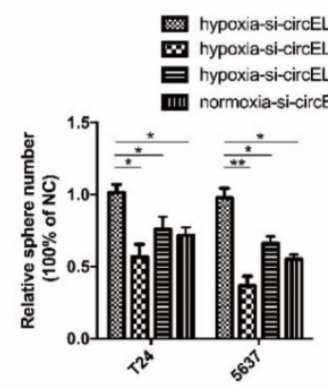

C

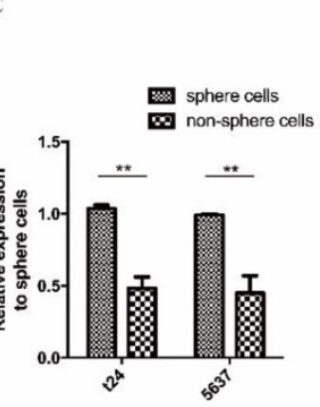

H

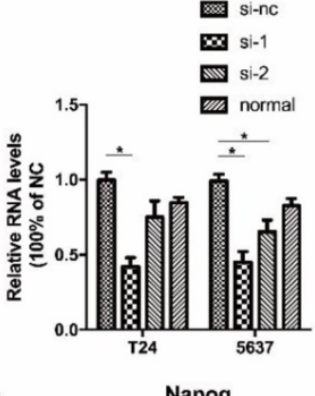

D

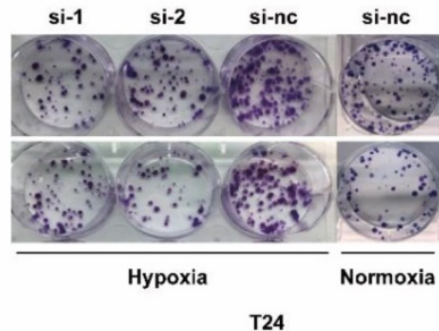

F

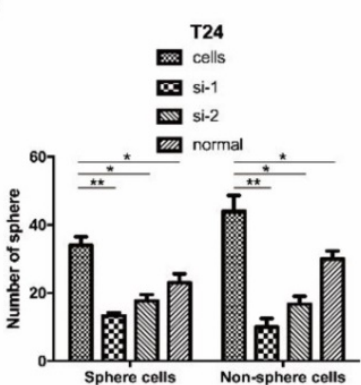

E

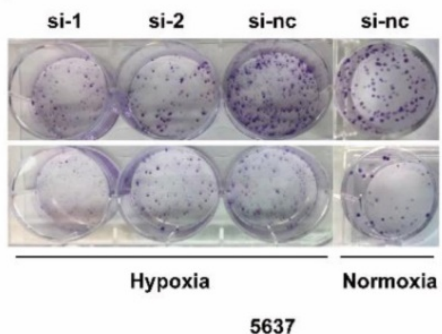

G

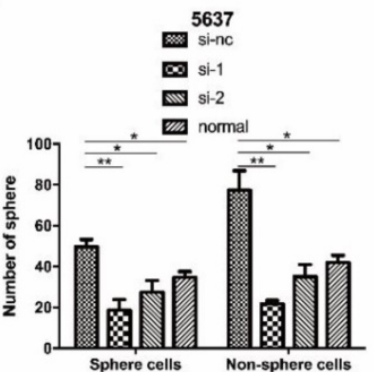

si-

initis

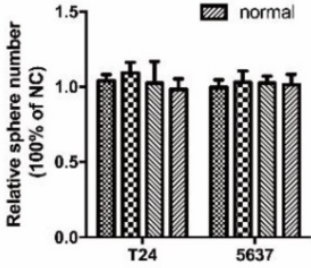

Oct-4

si-nc

mi $\mathrm{si}-1$

si-2

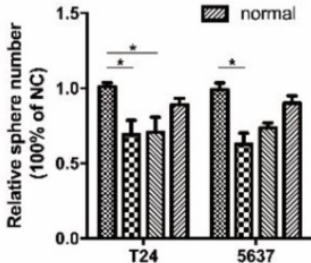

Figure 5: circELP3 may facilitate cisplatin resistance by targeting cancer stem-like cells. A: Sphere formation by T24 (the upper one) and 5637 cells (the lower one) was photographed microscopically at 40x; B: Spheres were counted under a light microscope; C: Quantitative real-time PCR was used to determine the expression of circELP3 between sphere-forming and non-sphere-forming cells; H, I, J: Quantitative real-time PCR was used to determine the expression of Sox2, Oct-4 and Nanog after treatment with siRNA for circELP3; D: Cell clone formation assays were performed to evaluate the effect of circELP3 on the self-renewal ability of sphere-forming cells and non-sphere-forming cells after treatment with $5 \mu \mathrm{M}$ cisplatin; $\mathrm{F}$ and $\mathrm{G}$ show the significant clone numbers in each treatment group. Scale bars: $\mathrm{A}, 100 \mu \mathrm{M}$.

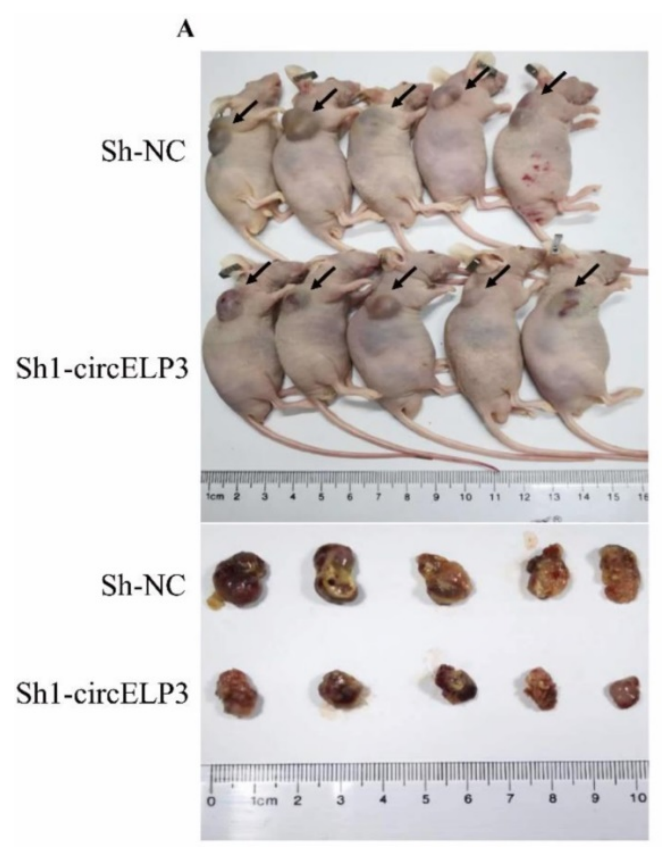

\pm Sh-NC

$\rightarrow$ Sh1-circELP3

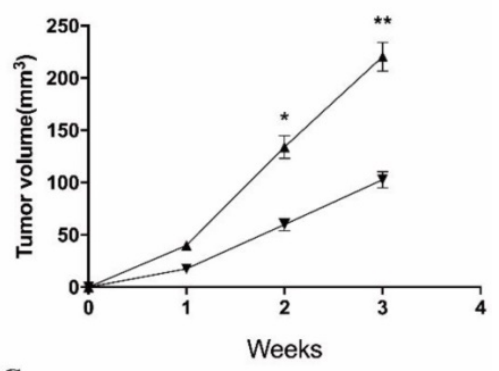

C

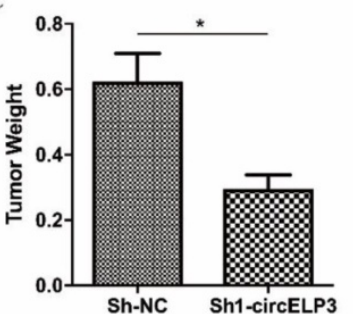

Figure 6: Interfering with circELP3 suppresses tumor xenograft growth in nude mice. A: Impairing circELP3 expression suppress the growth of bladder cancer in nude mice; B: volume of tumor xenografts was measured weekly, and the growth curve for the 2 groups is shown; C: The weights of tumor xenografts were measured 3 weeks after injection. 

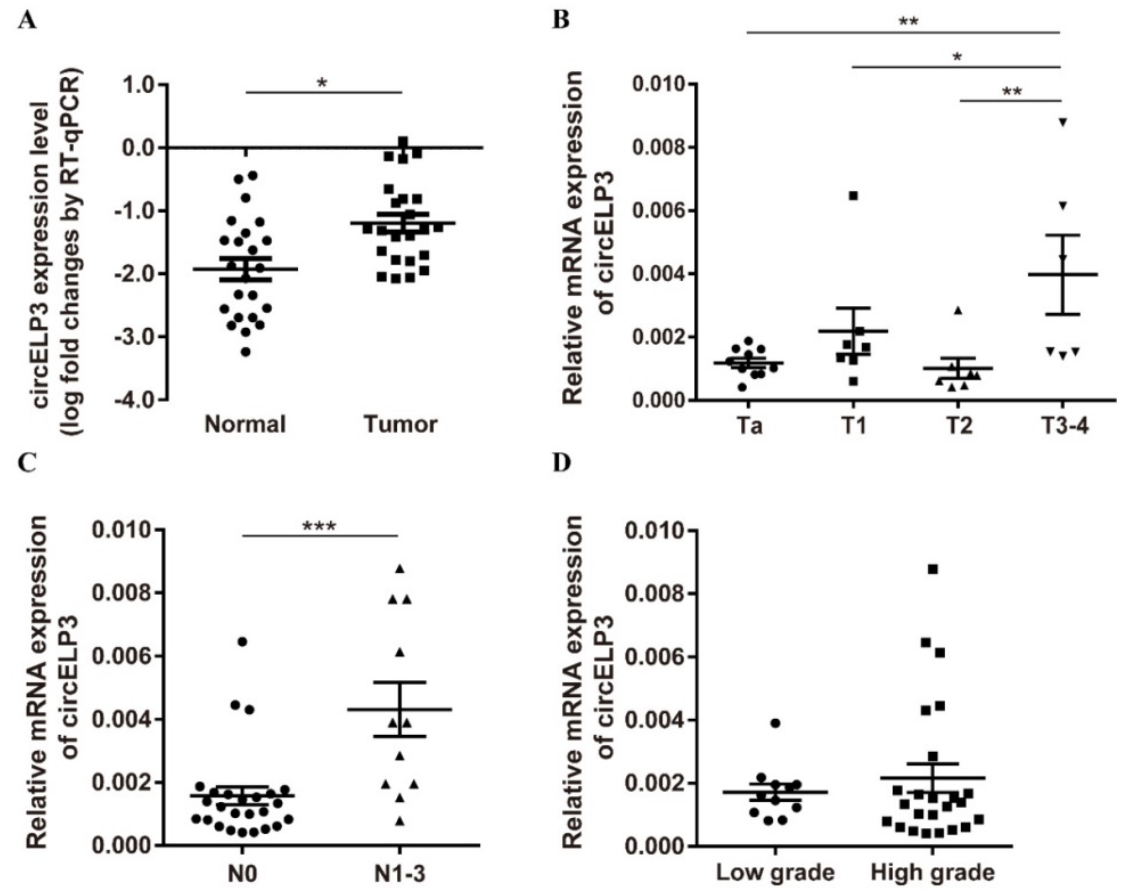

D

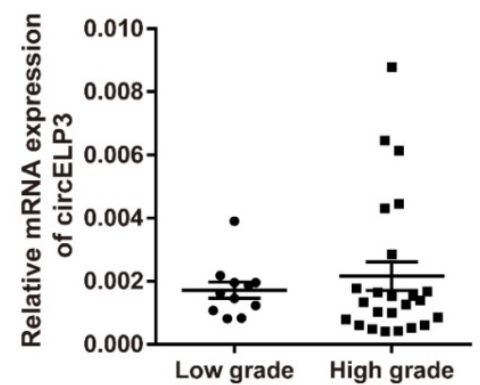

Figure 7: Clinical relevance of circELP3 in bladder cancer. A: Quantitative real-time PCR was used to detect the expression of circELP3 in 18 pairs of bladder cancer and normal tissues; B-D: Expression of circELP3 among 30 bladder cancer tissues. B shows differences between T stages; $\mathbf{C}$ shows differences between lymph node metastasis; $\mathbf{D}$ shows differences between tumor grades.

Cisplatin resistance is no more than a platitude in cancer research. In bladder cancer, cisplatin-based chemotherapy is the only clinical treatment if patients have progressed to advanced stage [23]. Hence, to some degree, we can say that cisplatin resistance shortens the survival of advanced bladder cancer patients. To address this drawback, cisplatin-based combined therapies are increasingly used in clinical bladder cancer treatment, such as cisplatin chemotherapy combined with a PD-L1 inhibitor [24]. Although some cases have shown improved clinical outcomes, drug resistance is still considered the major obstacle, and we only partially attribute this alleviation to immunity-related issues due to the PD-L1 inhibitor. Cisplatin is a platinum-based compound that can bind nucleotides to induce single-strand DNA breaks [25]. Damaged DNA then activates the DNA damage response to kill already unrepairable genomically damaged cells via apoptosis. Thus, research on cisplatin resistance has focused on the DNA damage response.

Hypoxia can cause DNA damage, and this DNA damage extraordinarily favors an adaptive response, promoting cancer progression. Previous reports have shown that hypoxia can enhance cisplatin resistance in cancer. High hypoxia levels in patient tissues are correlated with reduced outcomes for cisplatin-based chemotherapy and poor survival $[9,11]$. Although few reports have focused on demonstrating the mechanism between hypoxia and cisplatin resistance $[13,14]$, hypoxia does shed a new light on cisplatin resistance exploration. Here, we hypothesize that enhanced cisplatin resistance caused by hypoxia may lead to improved DNA repair capacity induced by the hypoxic adaptive response.

In this report, we found that hypoxia elevates circELP3 to partially facilitate a tumor promoting effect. It was found that under hypoxia; bladder cancer cell proliferation and drug resistance were greatly promoted compared with bladder cancer cells under normal oxygen conditions. Silencing circELP3 significantly reduced proliferation and cisplatin resistance of bladder cancer cells to the level that was measured for healthy bladder cancer cells under normal oxygen conditions. This effect further indicates the vital role of cirELP3 in hypoxia-induced tumor promotion. For bladder cancer, effective chemotherapy strongly relies on specifically and powerfully inducing cancer cell death, while apoptosis evasion and indefinite proliferation are the major phenotypes of drug resistance. Cancer stem cells are defined as cells that can self-renew and differentiate in cancer. By serving as seed cells in cancer, cancer stem cells are responsible for carcinogenesis, cancer growth, metastasis and relapse [26]. Despite their stronger self-renewal ability, better DNA damage repair is found in cancer stem cells [27]. Additionally, it has been reported that cancer stem-like cells contribute to cisplatin resistance in bladder cancer [4]. Hence, we are interested in whether hypoxia can facilitate cisplatin resistance by regulating cancer stem cells. 
We found that hypoxia enhances cisplatin resistance by elevating circELP3 expression in bladder cancer and activated circELP3 increases the portion of cancer stem-like cells in vitro. However, compared with the clone forming ability between sphere-forming cells and non-sphere-forming cells, there were no differences when cells were treated with siRNA for circELP3. The sphere-forming assay only demonstrates a higher proportion of cancer stem cells; the cell population in spheres is not strictly composed of cancer stem cells. We regard the cells as cancer stem-like cells. Hence, the effect of circELP3 on cancer stem cells could be interfered by the population of noncancer stem cells in the sphere. Further research is needed to isolate cancer stem cells more accurately and to verify the mechanism among circELP3, cisplatin and cancer stem cells.

RNA function strongly relies on its cellular location. Circular RNAs are classified as cytoplasmlocated or nuclear-located circular RNA [15]. As we mentioned previously, cytoplasm-located circular RNAs mainly work through ceRNA interaction, while nuclear-located circular RNAs are obligated to regulate protein coding or transcription. To furtherly evaluate the possible mechanism of circELP3 in bladder cancer, a florescence in situ hybridization assay was performed and showed that the circELP3 signal is mainly present in the cytoplasm. This result suggests that circELP3 may exert its role via ceRNA activity. Among various participants in the ceRNA regulatory network, miRNA sponge is the major player. Hence, circELP3 may exert its role by sponging multiples miRNAs. Through prediction on https://circinteractome.nia.nih.gov, several miRNAs are suggested. Among these miRNAs, miR-216 [28], miR-128 [29], miR-143 [30], miR-149 [31], miR-154 [32], miR-485 [33] have been shown to inhibit bladder cancer progression. Therefore, these predictions suggest that circELP3 may sponge those miRNAs to exert its biological regulation activity.

Although the mechanisms underlying the role of circELP3 in bladder cancer still require further study, we provide the first direct evidence that circular RNA participates in the adaptive response to hypoxia and plays a role in the progression and drug resistance of bladder cancer. We hope that more studies will focus on this RNA in the future, particularly to determine the regulation of cisplatin resistance by cancer stem cells, which will further reveal the mysteries of bladder cancer biology.

\section{Acknowledgments}

This project was supported by the National Natural Science Foundation of China (Grant No.
U1301221，81572514，81472384，81402106，81372729， 81272808).

\section{Competing Interests}

The authors have declared that no competing interest exists.

\section{References}

1. Cattrini C, Boccardo F. Atezolizumab and bladder cancer: facing a complex disease. Lancet. 2018; 391: 305-6.

2. Hurst $C D$, Knowles MA. Bladder cancer: Multi-omic profiling refines the molecular view. Nature reviews Clinical oncology. 2018; 15: 203-4

3. Wong YNS, Joshi K, Pule M, Peggs KS, Swanton C, Quezada SA, et al. Evolving adoptive cellular therapies in urological malignancies. The Lancet Oncology. 2017; 18: e341-e53.

4. Zhang Y, Wang Z, Yu J, Shi J, Wang C, Fu W, et al. Cancer stem-like cells contribute to cisplatin resistance and progression in bladder cancer. Cancer letters. 2012; 322: 70-7.

5. Owczarek TB, Kobayashi T, Ramirez R, Rong L, Puzio-Kuter AM, Iyer G, et al. ARF Confers a Context-Dependent Response to Chemotherapy in Muscle-Invasive Bladder Cancer. Cancer research. 2017; 77: 1035-46.

6. Pouyssegur J, Dayan F, Mazure NM. Hypoxia signalling in cancer and approaches to enforce tumour regression. Nature. 2006; 441: 437-43.

7. Mao X, Wong SY, Tse EY, Ko FC, Tey SK, Yeung YS, et al. Mechanisms through Which Hypoxia-Induced Caveolin-1 Drives Tumorigenesis and Metastasis in Hepatocellular Carcinoma. Cancer research. 2016; 76: 7242-53.

8. Brahimi-Horn MC, Chiche J, Pouyssegur J. Hypoxia and cancer. Journal of molecular medicine. 2007; 85: 1301-7.

9. Vaupel P, Mayer A. Hypoxia in cancer: significance and impact on clinical outcome. Cancer metastasis reviews. 2007; 26: 225-39.

10. Bristow RG, Hill RP. Hypoxia and metabolism. Hypoxia, DNA repair and genetic instability. Nature reviews Cancer. 2008; 8: 180-92.

11. Wohlkoenig C, Leithner K, Deutsch A, Hrzenjak A, Olschewski A, Olschewski $\mathrm{H}$. Hypoxia-induced cisplatin resistance is reversible and growth rate independent in lung cancer cells. Cancer letters. 2011; 308: 134-43.

12. Sun XP, Dong X, Lin L, Jiang X, Wei Z, Zhai B, et al. Up-regulation of survivin by AKT and hypoxia-inducible factor 1alpha contributes to cisplatin resistance in gastric cancer. The FEBS journal. 2014; 281: 115-28.

13. Chang HY, Chang TC, Huang WY, Lee CT, Yen CJ, Tsai YS, et al. RON Nuclear Translocation under Hypoxia Potentiates Chemoresistance to DNA Double-Strand Break-Inducing Anticancer Drugs. Molecular cancer therapeutics. 2016; 15: 276-86.

14. Liu Y, Bernauer AM, Yingling CM, Belinsky SA. HIF1alpha regulated expression of XPA contributes to cisplatin resistance in lung cancer. Carcinogenesis. 2012; 33: 1187-92.

15. Kristensen LS, Hansen TB, Veno MT, Kjems J. Circular RNAs in cancer: opportunities and challenges in the field. Oncogene. 2018; 37: 555-65.

16. Yang C, Yuan W, Yang X, Li P, Wang J, Han J, et al. Circular RNA circ-ITCH inhibits bladder cancer progression by sponging miR-17/miR-224 and regulating p21, PTEN expression. Molecular cancer. 2018; 17: 19

17. Li Y, Zheng F, Xiao X, Xie F, Tao D, Huang C, et al. CircHIPK3 sponges miR-558 to suppress heparanase expression in bladder cancer cells. EMBO reports. 2017; 18: 1646-59.

18. Zhong Z, Huang M, Lv M, He Y, Duan C, Zhang L, et al. Circular RNA MYLK as a competing endogenous RNA promotes bladder cancer progression through modulating VEGFA/VEGFR2 signaling pathway. Cancer letters. 2017; 403: 305-17.

19. Yu J, Xu QG, Wang ZG, Yang Y, Zhang L, Ma JZ, et al. Circular RNA cSMARCA5 inhibits growth and metastasis in hepatocellular carcinoma. Journal of hepatology. 2018; 68: 1214-27.

20. Koelwyn GJ, Quail DF, Zhang X, White RM, Jones LW. Exercise-dependent regulation of the tumour microenvironment. Nature reviews Cancer. 2017; 17: 620-32.

21. Huo D, Liu S, Zhang C, He J, Zhou Z, Zhang H, et al. Hypoxia-Targeting, Tumor Microenvironment Responsive Nanocluster Bomb for Radical-Enhanced Radiotherapy. ACS nano. 2017; 11: 10159-74.

22. Choudhry H, Harris AL. Advances in Hypoxia-Inducible Factor Biology. Cell metabolism. 2018; 27: 281-98.

23. Liu D, Abbosh P, Keliher D, Reardon B, Miao D, Mouw K, et al. Mutational patterns in chemotherapy resistant muscle-invasive bladder cancer. Nature communications. 2017; 8: 2193.

24. Lopez-Beltran A, Cheng L, Montorsi F, Scarpelli M, Raspollini MR, Montironi R. Concomitant bladder cancer and prostate cancer: challenges and controversies. Nature reviews Urology. 2017; 14: 620-9.

25. Zamble DB, Lippard SJ. Cisplatin and DNA repair in cancer chemotherapy. Trends in biochemical sciences. $1995 ; 20: 435-9$.

26. Ayob AZ, Ramasamy TS. Cancer stem cells as key drivers of tumour progression. Journal of biomedical science. 2018; 25: 20.

27. Blanpain C, Mohrin M, Sotiropoulou PA, Passegue E. DNA-damage response in tissue-specific and cancer stem cells. Cell stem cell. 2011; 8: 16-29. 
28. Xiao J, Lin HY, Zhu YY, Zhu YP, Chen LW. MiR-126 regulates proliferation and invasion in the bladder cancer BLS cell line by targeting the PIK3R2-mediated PI3K/Akt signaling pathway. OncoTargets and therapy. 2016; 9: 5181-93.

29. Zhou XU, Qi L, Tong S, Cui YU, Chen J, Huang T, et al. miR-128 downregulation promotes growth and metastasis of bladder cancer cells and involves VEGF-C upregulation. Oncology letters. 2015; 10: 3183-90.

30. Wang H, Li Q, Niu X, Wang G, Zheng S, Fu G, et al. miR-143 inhibits bladder cancer cell proliferation and enhances their sensitivity to gemcitabine by repressing IGF-1R signaling. Oncology letters. 2017; 13: 435-40.

31. Sugawara S, Yamada Y, Arai T, Okato A, Idichi T, Kato M, et al. Dual strands of the miR-223 duplex (miR-223-5p and miR-223-3p) inhibit cancer cell aggressiveness: targeted genes are involved in bladder cancer pathogenesis. Journal of human genetics. 2018; 63: 657-68.

32. Zhao X, Ji Z, Xie Y, Liu G, Li H. MicroRNA-154 as a prognostic factor in bladder cancer inhibits cellular malignancy by targeting RSF1 and RUNX2. Oncology reports. 2017; 38: 2727-34.

33. Chen Z, Li Q, Wang S, Zhang J. miR4855p inhibits bladder cancer metastasis by targeting HMGA2. International journal of molecular medicine. 2015; 36: $1136-42$ 